\title{
Thank You Peer Reviewers
}

Peer review is the cornerstone of the mission of ASHS journals - to advance the science of horticulture by publishing accurate, clear, reproducible research. Reviewers are the unsung heroes of that process.

To that end, we wish to express sincere appreciation to the scientists who have generously contributed their valuable time and expertise to assist both authors and editors in maintaining the high quality of the Journal of the American Society for Horticultural Science.

The individuals listed below have provided reviews for one or more manuscripts during the past publication year. Their names are published here in recognition of their valuable contributions to ASHS and horticultural science. Every effort has been made to compile an accurate list from the records kept by the Society; any omissions are inadvertent.

\begin{tabular}{|c|c|c|c|}
\hline Lisa Alexander & Zhanao Deng & David Jones & Ahmad A. Omar \\
\hline Teresita D. Amore & Jose V. Die-Ramon & Wayne M. Jurick II & Olaya Pérez-Tornero \\
\hline Francisco Romário Andrade & John M. Dole & Rina Kamenetsky Goldstein & Dilip R. Panthee \\
\hline Figueiredo & Ashraf El-Kereamy & Chahine Karmous & Dan E. Parfitt \\
\hline Hamid Ashrafi & Choaa El-Mohtar & Mary Beth Kirkham & H. Brent Pemberton \\
\hline Jinhe Bai & Shenghua Fan & Joshua D. Klein & Yingshu Peng \\
\hline Peter J. Balint-Kurtie & Yanping Fan & Chieri Kubota & Penelope Perkins-Veazie \\
\hline John Bamberg & Jorge M. Fonseca & Anuj Kumar & Pere Puigdomenech \\
\hline Ekaterina N. Baranova & Jonathan M. Frantz & Madhurababu Kunta & Erja Rappe \\
\hline Grzegorz Bartoszewski & Anne Frary & Benoît Lacombe & David R. Rudell \\
\hline Randolph M. Beaudry & Darla French & Brian Leckie & Uttara C. Samarakoon \\
\hline Vidmantas Bendokas & Qin $\mathrm{Fu}$ & Jungmin Lee & German Sandoya \\
\hline Andre Berville & Tongcheng Fu & Gene E. Lester & Carolyn Francis Scagel \\
\hline Guillaume Besnard & Yinbo Gan & Deying Li & Babar Shahzad \\
\hline Gehendra Bhattarai & Ksenija Gasic & Tongyin Li & Qingsong Shao \\
\hline Ruchika Bhawal & Eduardo Girardi & Zhenchang Liang & Yunyan Sheng \\
\hline Paolo Boccacci & Christopher Charles Gottschalk & Yuexue Liu & Supaart Sirikantaramas \\
\hline Martin O. Bohn & Thomas M. Gradziel & Tomas Lobos & Guo-qing Song \\
\hline David Hawkins Byrne & Wenwu Guo & Eliezer S. Louzada & Timothy M. Spann \\
\hline Tina M. (Waliczek) Cade & Michael J. Havey & Christopher A. Lowry & Rolston St. Hilaire \\
\hline Zejiang Cai & Ryan J. Hayes & Qian Ma & John R. Stommel \\
\hline Ann M. Callahan & Junna He & Luis Maas & Youping Sun \\
\hline Linda Chalker-Scott & Richard J. Heerema & Subas Malla & Jon Y. Suzuki \\
\hline Chun-Yen Chang & Elizabeth Henry & Nathan Maren & Cassandra L. Swett \\
\hline Dario J. Chavez & Peter M. Hirst & James P. Mattheis & Daocheng Tang \\
\hline Jianjun Chen & Patricia S. Holloway & Thomas E. Michaels & Lisa Tang \\
\hline Fangyun Cheng & Bo Hong & Milica Milutinović & Christian Tobias \\
\hline Karen Ann Cichy & Longxing $\mathrm{Hu}$ & Takayuki Mizuno & Darren H. Touchell \\
\hline Thomas A. Colquhoun & Samuel Forrest Hutton & Ramon Molina-Bravo & Moneesh Upmanyu \\
\hline Patrick J. Conner & Massimo Iorizzo & Thomas J. Molnar & Allen van Deynze \\
\hline Ryan N. Contreras & Robert Lawrence Jarret & Om Narayan & Tripti Vashisth \\
\hline Peter S. Cousins & Cai-Zhong Jiang & Jianxin Niu & Gayle M. Volk \\
\hline Angel R. Del Valle Echevarria & Yiwei Jiang & Gerardo H. Nunez & Phillip Andrew Wadl \\
\hline
\end{tabular}




$\begin{array}{llll}\text { Marisa M. Wall } & \text { David Wolyn } & \text { Xiaoming Yang } & \text { Xiaonan Yu } \\ \text { Kellie J. Walters } & \text { Glenn C. Wright } & \text { Raymond K. Yokomi } & \text { Donglin Zhang } \\ \text { Jun-E Wang } & \text { Jian Wu } & \text { Gad G. Yousef } & \text { Xunzhong Zhang } \\ \text { Yiling Wang } & \text { Xingbo Wu } & \text { Cliff Runze Yu } & \text { Jun Zheng } \\ \text { Yin-Tung Wang } & \text { Yan Xu } & \text { Jiali Yu } & \text { Xing-Wei Zheng } \\ \text { Ryan M. Warner } & \text { Jun Yang } & \text { Jingwei Yu } & \text { David C. Zlesak } \\ \text { Vance McFadyen Whitaker } & \text { Tianbao Yang } & \text { Ping Yu } & \end{array}$

